\title{
Genetic Programing for Topological Investigation of Optimum Eccentricity of EBF Systems
}

\author{
Hassanali Mosalman Yazdi, M. Mosalman, F. Mosalman Yazdi, and A. Mosalman Yazdi
}

\begin{abstract}
Eccentrically Braced Frame (EBF) systems are one of the most common types of steel braced systems. These systems are used for designing structures against the lateral loads. For designing these systems, determination the best connection location with conventional methods is not trivial. Hence, in this paper, a method based on Genetic programming is proposed to investigate topologically all feasible connection points in the panel area. In this topological evaluation, eccentricity of different locations along with a few designing criteria are considered. These criteria which so-called objective functions are composed to conduct a single function. Finally, genetic modeling is proposed to go through searching the best eccentricity for the system. The results of the proposed method are so precise and trustful.
\end{abstract} load.

Index Terms-Brace, eccentricity, genetic algorithm, Seismic

\section{INTRODUCTION}

In regions subjected to seismic activities, the occurrence of severe earthquakes can cause serious damage to properties and loss of lives if buildings are not provided with seismic resistance capability. The need to have buildings suitably designed against earthquake is even more necessary in highly urbanized areas with many tall buildings, where economical and human losses are major concern to the community.

The current seismic design philosophy has as its first consideration the safety of lives in the event of a severe earthquake. As the nature and occurrence of earthquakes are indeterministic, it is necessary to consider different levels of earthquakes intensity in the design of earthquake resistance structures. These requirements are expressed in three levels of structural performance (UBC,1997) as follows:

- The structure is to resist minor earthquakes without damage.

- The structure should resist moderate and frequently occurring earthquakes without structural damage, but limited non-structural damage may be tolerated.

- The structure should not collapse under severe earthquakes, where life safety must be maintained.

Exceptions to these requirements are important/critical facilities such as hospitals, fire stations, nuclear centres and other facilities where more stringent and critical requirements should be followed.

The provisions permit structures to yield under strong

Manuscript received December 15, 2012; revised March 10, 2013.

Hassanali Mosalman Yazdi is with the Faculty of Engineering, Maybod Branch, Islamic Azad University, Maybod, Iran (e-mail: hmosalman@gmail.com).

M. Mosalman, F. Mosalman Yazdi, and A. Mosalman Yazdi are with the Maybod Branch, Islamic Azad University, Maybod, Iran. earthquake loadings as it is generally uneconomical to design structures to remain elastic under severe earthquakes. The ability of structures to sustain loads into the plastic and strain hardening regions provide an economical solution for seismic design under severe earthquake loading.

Generally, steel structures are used extensively for medium and high rise buildings due to their excellent strength and ductility properties. Mostly steel structures are designed to resist the lateral load by using brace elements.

Eccentrically Brace Frame (EBF) and Concentric Brace Frame (CBF) are two common types of braced systems. EBF system performs better than the CBF system against seismic loads. This system has a very good energy dissipation capacity and is stable against periodic loads.

Several possible types of EBFs are illustrated in Fig. 1 with different configurations such as D-brace frame, K-brace frame, and V-brace frames respectively shown.

In all such frames, the vertical components of axial forces in the braces are held in equilibrium by shear and bending moments in short beams of length e, which are called active links. These active links, as shown in Fig. 2, are designed to remain elastic at working loads and deformed inelastically due to overloading, thereby dissipating large amount of energy. In this system the hazardous brace buckling can be entirely prevented since the link acts as fuse to limit the brace axial force. Also, this frame has a much greater lateral resisting capacity than that of an MRF if the beam sections used are the same.
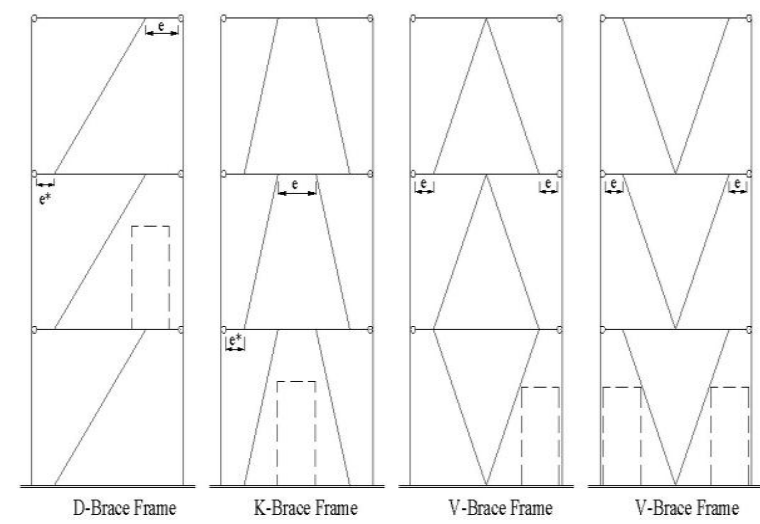

Fig. 1. Various types of EBFs [1]

However for designing this system whatever is so important, is the location of connection point or in the other word, the length of active beam. This element as mentioned before has a significant effect on the stiffness of system thereby some parameters such as the cross section of brace elements, dimensions of openings and frame and the location 
of the connection affect the system's stiffness. With consideration these parameters recognising the best length for the active beam or the best eccentricity for the system which has the highest stiffness and lowest weight of brace elements is not trivial and more designers use trail and error to get through this selection. Not trustworthy connection point selection and so much time consuming are characteristics of the conventional methods.

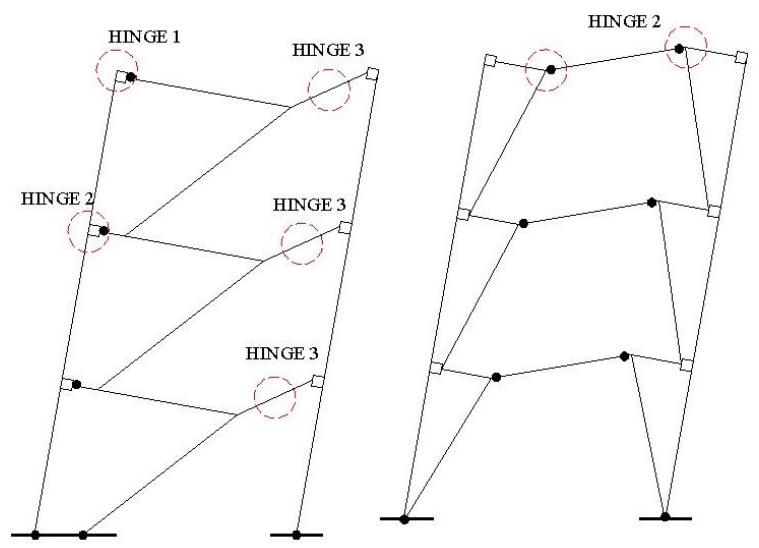

Fig. 2. Energy dissipation mechanism of EBFs [2]

In this paper a method based on multi-objective genetic algorithm is proposed to help designers in the selection of the best connection point. In this proposed method the stiffness equation of a particular type of EBF system which is called Off-Centre Brace (OCB) is obtained parametrically and along with the weight of brace elements. Both of these parametric formulas are as the fitness function. In this paper the eccentricity optimization is done by multi objective genetic algorithm which is based a modified plain aggregate method by designer's coefficient. Eventually, after analytical studies a MATLAB computer programming is used in order to do the calculations.

\section{Genetic Algorithm}

Genetic algorithms (GAs) are search and optimization tools, which work differently, compared to classical search and optimization methods. This search technique is based on the principals of genetics and natural selection, at first initiated by Holand in the 1970s [3]. Because of their broad applicability, ease of use, and global perspective, GAs have been increasingly applied to various search and optimization problems in the recent past.

GAs are initialised with a population of guesses. These are usually random and will be spread throughout the search space. Typically, these initial guesses are held as binary encodings of the true variables [4], [5]. In the other word encoding is the first operation in a GA. Each variable is represented by using a bit string which is merged to form a chromosome that represents a design. The elements of the string corresponds to genes and the values those genes can take to alleles. During each generation, each individual in the population is evaluated using the fitness function [6]. Genetic operators are applied to the individuals of the population in order to generate the next generation of such individuals. The procedure continues until the termination condition is

satisfied. A typical algorithm uses three operators, selection, crossover and mutation to direct the population towards convergence at the global optimum.

However this initial population is then processed by the three main operators [7], [8].

\section{A. Crossover}

Crossover exchanges the chosen portions of two parents and generates the new individuals. These chosen portions are decided by the randomly chosen crossover points. One of the commonly used crossover operations is one-point crossover which is used in this paper (see Fig. 3 ). The crossover operator roughly mimics biological recombination between two single chromosomes.

\section{parents \begin{tabular}{|l|l|l|l|l|l|l|l|l|l|l|l|l|l|l|l|l|l|}
\hline 0 & 0 & 0 & 0 & 0 & 0 & 0 & 0 & 0 & 0 & 0 & 0 & 0 & 0 & 0 & 0 & 0 & 0 \\
\hline
\end{tabular} \begin{tabular}{|l|l|l|l|l|l|l|l|l|l|l|l|l|l|l|l|l|l|}
\hline 1 & 1 & 1 & 1 & 1 & 1 & 1 & 1 & 1 & 1 & 1 & 1 & 1 & 1 & 1 & 1 & 1 & 1 \\
\hline
\end{tabular} \begin{tabular}{|l|l|l|l|l|l|l|l|l|l|l|l|l|l|l|l|l|l|}
\hline 0 & 0 & 0 & 0 & 0 & 1 & 1 & 1 & 1 & 1 & 1 & 1 & 1 & 1 & 1 & 1 & 1 & 1 \\
\hline
\end{tabular} children \begin{tabular}{|l|l|l|l|l|l|l|l|l|l|l|l|l|l|l|l|l|l|}
\hline 1 & 1 & 1 & 1 & 1 & 0 & 0 & 0 & 0 & 0 & 0 & 0 & 0 & 0 & 0 & 0 & 0 & 0 \\
\hline
\end{tabular}}

Fig. 3. One point crossover operator

\section{B. Mutation}

Mutation is a random alteration of a string that produces incremental changes in the offspring generated through crossover. By itself, mutation is equivalent to a random search. However, in GA, mutation also helps to prevent premature convergence(see Fig. 4).

\begin{tabular}{l|l|l|l|l|l|l|l|l|l|l|l|l|l|l|l|l|l|l|} 
parent & 1 & 1 & 1 & 1 & 1 & 1 & 1 & 1 & 1 & 1 & 1 & 1 & 1 & 1 & 1 & 1 & 1 & 1 \\
\hline
\end{tabular}
child

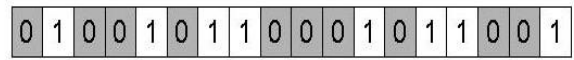

Fig. 4. Mutation operator on the survival individual and on the offspring

\section{Selection}

This operator selects chromosomes (individuals) in the population for reproduction. The fitter the chromosome, the more times it is likely to be selected to produce. In the other word a selection scheme determines the probability of an individual being selected for producing offspring by crossover and mutation. In order to search for increasingly better individuals, fitter individuals should have higher probabilities of being selected while unfit individuals should be detected only with small probabilities.

In this research the Ranked based selection method is used. Rank-based selection does not calculate selection probabilities from fitness values directly. It sorts all individuals according to their fitness values first and then computes selection probabilities according to their ranks rather than their fitness values. Hence rank-based selection can maintain a constant selection pressure in the evolutionary search and avoid some of the problems encountered by roulette wheel selection. There are many different rank-based selection schemes. Several are introduced below: 
Assume the best individual in a population ranks first. The probability of the selection of individual $i$ can be calculated as follows (Baker,1985):

$$
p_{i}=\frac{1}{\mu}\left(\eta_{\max }-\left(\eta_{\max }-\eta_{\min }\right) \frac{i-1}{\mu-1}\right)
$$

where $\mu$ is the population size, $\eta_{\max }$ is the probability of the best individual, $\eta_{\min }$ is the probability for the worst individual. Intermediate individuals' ranks are decreased from $\eta_{\max }$ to $\eta_{\min }$ proportionally to their rank. Setting $\eta_{\text {min }}=0$, the maximum selective pressure is obtained.

A rank-based selection scheme with a stronger selection pressure is the following nonlinear ranking scheme (Yao, 1993).

$$
p_{i}=\frac{\mu+1-i}{\sum_{j=1}^{\mu} j}
$$

The exponential function has the following form (Michalewicz, 1996).

$$
p_{i}=\frac{\eta(1-\eta)^{i-1}}{c}
$$

where $c$ is the normalization factor chosen so that the sum of the probabilities is unity. In this case a larger value of $\eta$ implies [9], [10].

\section{Terminating Criterion}

Two terminating criteria are utilized in this study. The first one considers a pre-selected threshold for the fitness values in generation, in which if the highest fitness value exceeds the threshold, the process will be terminated. The other condition is a pre-selected limit on the number of generations. If the number of generations reaches this limit, the process is stopped. In either case, that solution in the last generation which has the highest fitness value is selected as the resulting optimal solution.

\section{E. Multi Criteria Combination}

Search problems encountered in the real world area often characterized by the fact that many objectives must be satisfied. Although this topic is called multi-objective optimization.

Conventional optimization techniques, such as gradient-based and simplex-based methods, were not designed to cope with multiple-objectives search problems, which have to be transformed into single objective problems prior to optimization. On the other hand, evolutionary algorithms are considered to be better tailored to multiple-objectives optimization problems. This is mainly due to the fact that multiple individuals are sampled in parallel, and the search for multiple solutions can be more effective briefly and also can say the multi-objective optimization is the area where evolutionary computation really distinguishes from its competitors [9, 10]. For instance, in this paper there are two fitness functions, weight and system's stiffness. The aim is to obtain the best connection point that satisfies both of these function with having maximum stiffness and minimum weight.

However, in this paper plain aggregation approach which is on of the evolutionary computing techniques is used and it was modified to interpret the designer decisions.

A plain Aggregation approach is the most straightforward approach, to transform the objective vector in a scalar.

Sum, i.e.,

$$
f(x)=\sum_{i=1}^{n} w_{i} f_{i}(x) \quad \text { where } \sum_{i=1}^{k} w_{i}=1
$$

According to the above equation, the fitness value of the individual $x$ will be given by the sum, over $\mathrm{n}$ objectives, of the fitness corresponding to each objective, $f_{i}(x)$, multiplied by the weight $w_{i}$.

For numerical calculation, regarding this proposal method two objective functions are used as stiffness and weight of OCB system which are as follows [11]-[13]:

$$
K=\frac{n L^{2} E A}{\left((n H)^{2}+(L(1-m))^{2}\right)^{\frac{1}{2}}} \times \frac{m n}{\left(\left(m-m^{2}\right) L^{2}+\left(n-n^{2}\right) H^{2}\right)}
$$

$$
W_{T}=\rho \sum_{i=1}^{3} A_{i} L_{i}=\rho A_{1}\left(L_{1}+\frac{n L_{2}^{2}}{m L}+\frac{(1-(m+n)) L_{3}}{m L_{1}}\right)
$$

In order to modeling the eccentricity of this system two parameters, $m$ and $n$ which are coefficients of width and height of frame respectively, are introduced. $E, L$ and $A$ are respectively module of elasticity and cross section area of brace member.

\section{RESULTS AND DISCUSSION}

To topologically investigate the proposed approach for determining of the best connection location or optimal eccentricity, the stiffness and the weight of all possible point of different $m$ and $n$ in the feasible area of a frame with above characteristics are calculated. The opening dimensions of this example considered as height to width is 2.5 to 2 meter. The results of different feasible values of $m$ and $n$ are calculated based on objective functions are shown in Table $\mathrm{I}$ as a coefficient of EA and $A \rho$, respectively, where, $A$ is the cross section of element one.

For this particular configuration, it is shown that the stiffness of the best selection for the connection point is equal to $0.058065 \mathrm{EA}$, which is located in a point with a distance of $X=2$ and $Y=1.5$ meter from top right corner of the frame.

Now, go through examining proposed approach which is a program based on genetic algorithm with considering different population size, mutation rate, number of bits for each variable and selection rate, the results show a very good convergence and high precision on determination of the 
connection point. The output indicates that the best connection point is located at the coordinate of $\mathrm{X}$ and $\mathrm{Y}$ equal to 1.9994 and 1.5004 meter respectively. At this point, the criteria do not have any privilege. Hence, the result calculated the stiffness and weight are found as 0.0581EA and 48.671A ton, respectively.

This gives very accurate results compared to conventional method. It can be concluded that the optimization based on multi objective genetic algorithm program is reliable due to accuracy and efficiency of this method.

\begin{tabular}{|c|c|c|c|c|c|c|c|c|}
\hline $\mathrm{m}$ & $\begin{array}{l}\text { Fitness } \\
\text { Function }\end{array}$ & 0.1 & 0.2 & 0.3 & 0.4 & 0.5 & 0.6 & 0.7 \\
\hline \multirow{2}{*}{0.1} & Stiffness & 0.0015338 & 0.0024807 & 0.0035355 & 0.0049818 & 0.0072102 & 0.011024 & 0.014061 \\
\hline & Weight & 7.9511 & 6.2017 & 5.6569 & 5.4252 & 5.3344 & 5.3358 & 5.3676 \\
\hline \multirow{2}{*}{0.2} & Stiffness & 0.0043386 & 0.0075792 & 0.011137 & 0.015811 & 0.022646 & 0.03354 & \\
\hline & Weight & 10.8465 & 7.7611 & 6.7765 & 6.3246 & 6.0908 & 5.9628 & \\
\hline \multirow{2}{*}{0.3} & Stiffness & 0.0079057 & 0.014235 & 0.021103 & 0.029814 & 0.041943 & & \\
\hline & Weight & 12.6491 & 8.661 & 7.3532 & 6.7082 & 6.3111 & & \\
\hline \multirow{2}{*}{0.4} & Stiffness & 0.012307 & 0.022361 & 0.033076 & 0.046225 & & & \\
\hline & Weight & 13.4026 & 8.9443 & 7.4421 & 6.6564 & & & \\
\hline \multirow{2}{*}{0.5} & Stiffness & 0.017855 & 0.032317 & 0.047384 & & & & \\
\hline & Weight & 13.2095 & 8.692 & 7.1299 & & & & \\
\hline \multirow{2}{*}{0.6} & Stiffness & 0.025214 & 0.045 & & & & & \\
\hline & Weight & 12.2034 & 8 & & & & & \\
\hline
\end{tabular}

\section{CONClusions}

Genetic programming can be claimed as a one of the best method for topological optimization for determination of the best connection location for EBF system. In this paper a method based on Evolutionary algorithms is proposed. The results indicate that the proposed method is so précis and can be used by designers. This method is a good replacement for the conventional method for determination the connection point of EBF system which is mostly selected by trial and error method. Using this method can facilitate the designing procedures of this system.

\section{REFERENCES}

[1] I. F. Khatib, S. A. Mahin, and K. S. Pister, Seismic behavior of concentrically braced steel frames, Earthquake Engineering Research Center (UCB/EERC-88/01), University of California: Berkeley, CA, 1988.

[2] K. Kasai and P. E. P., A study of seismically resistant eccentrically braced steel frame systems, UCB/EERC-86/01. p. (PB87 124 178/AS)A14, 1986.

[3] J. Holland, Adaptation in Natural and Artificial Systems, Ann Arbor, MI:The University of Michigan Press, 1975.

[4] M. Mitchell, An Introduction to Genetic Algorithms, MIT Press, 1996.

[5] E. L. Lawler, Combinatorial optimization: networks and matroids, New York: Holt, Rinehart and Winston, 1976.

[6] H. A. M. Yazdi and N. H. R. Sulong, "Optimization of Off-Centre bracing system using Genetic Algorithm," Journal of Constructional Steel Research, vol. 67, no. 10, pp. 1435-1441, 2011.
[7] R. L. Haupt and S. E. Haupt, Practical Genetic Algorithms, second ed. John Wiley \&. Sons, 2004.

[8] H. A. M. Yazdi and N. H. R. Sulong, "Genetic algorithm in locating the optimum mid-connection of Off-Centre braced system," Structure and Infrastructure Engineering, 2012, pp. 1-10.

[9] R. S. Zebulum, M. A. Pacheco, and M. M. B. R. Vellasco, Evolutionary Electronics: Automatic Design of Electronic Circuits and Systems by Genetic Algorithms, CRC Press, 2002, pp. 299.

[10] M. Sakawa, Genetic Algorithms and fuzzy multi-objective optimization, Academic Publishers, 2002.

[11] A. Mosalman and N. H. Ramli sulong, "Eccentricity Optimization of NGB System by Using Multi-Objective Genetic Algorithm," Journal of Applied Sciences, vol. 9, no. 19, pp. 3502-3512, 2009.

[12] H. Saffari and H. M. Yazdi, "An efficient and direct method for out-of-plane buckling analysis of Y-braced steel frames," Journal of Constructional Steel Research, vol. 66, no. 8-9, pp. 1107-1111.

[13] H. Mosalman and N. R. Sulong, "Parametric investigation on an off-centre braced frame system's stiffness," International Journal of the Physical Sciences, vol. 5, no. 17, pp. 2642-2651, 2010.

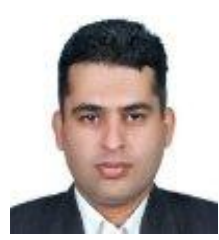

Hassanali Mosalman Yazdi is as assistant professor at Maybod Branch, Islamic Azad University. His research interest is application of Artificial Intelligence in structural optimizations. He was a Post Doctoral research fellow at university of Malaya.

M. Mosalman is a member of faculty at Maybod branch, Islamic Azad University.

F. Mosalman Yazdi and A. Mosalman Yazdi are members of faculty at Mehriz Branch Islamic Azad University, Mehriz, Iran. 